\title{
Consumo de alcohol y drogas en adolescentes de la provincia de Santa Elena
}

\section{Alcohol and drug consumption in adolescents province of Santa Elena}

\author{
Nancy Domínguez Rodríguez*1 ${ }^{1}$, Elsie Saavedra Alvarado ${ }^{1}$, Bolívar Suárez Lindao ${ }^{1}$, Anabel Sarduy Lugo ${ }^{1}$ \\ ${ }^{1}$ Universidad Estatal Península de Santa Elena. Ecuador \\ *ndominguez@upse.edu.ec
}

DOI: https://doi.org/10.26871/killkana_salud.v4i3.691

\begin{abstract}
Resumen
Contexto: El consumo de drogas en la actualidad es un importante fenómeno social que afecta especialmente a los adolescentes. La percepción de riesgo y los factores de riesgo, junto al ocio, el tiempo libre y la vida recreativa, son elementos que se deben considerar para comprender esta problemática, la práctica de programas adecuados y eficaces de prevención y tratamiento contribuye a su tratamiento. Objetivo: Determinar la percepción que tienen los adolescentes de la provincia Santa Elena en relación con el consumo de drogas y alcohol. Metodología: Los datos provienen del cuestionario ASSIST V3.0 OMS, dirigido a los adolescentes del Colegio la Libertad de los bachilleratos diurnos y vespertinos en un total de 431 encuestados. El diseño de la muestra fue probabilístico, estratificado y por conglomerados en varias etapas de muestreo. Resultados: el $34 \%$ de los adolescentes encuestados se inclinan al consumo de alcohol, el uso de bebidas alcohólicas son las sustancias que de mayor uso en 142 encuestados, mientras que el consumo de tabaco 55 de ellos lo realizan, donde mencionan que dicho consumo es concerniente a los problemas intrafamiliares presentes en cada hogar, a diferencia del rutina de cannabis coligado a la ansiedad o eventos de hiperactividad que pueden ser punto de partida para mayores complicaciones en la salud de los adolescentes. Conclusiones: El entorno que rodea a los jóvenes de 12 a 17 años indica que el consumo de drogas está cada vez más presente. Los índices de consumo se han incrementado en la provincia de Santa Elena donde nace la necesidad de desarrollar campañas de promoción y prevención frente a este fenómeno social.
\end{abstract}

Palabras clave: Adolescentes, Consumo, Drogas, Percepción, Salud.

\begin{abstract}
Drug use today is an important social phenomenon that especially affects adolescents, given the high availability, making decisions for not using or abstinence from these. The perception of risk and risk factors, along with leisure, free time and recreational life, are elements that we must consider understanding this problem, the practice of adequate and effective prevention and treatment programs contributes to its treatment. Objective: To determine the perception that adolescents have in the Santa Elena province in relation to drug and alcohol consumption. Methodology: The data come from the ASSIST V3.0 WHO questionnaire, aimed at adolescents from College la Libertad of the day and evening high schools in 431 respondents. The sample design was probabilistic, stratified and by conglomerates in various stages of sampling. Results: $34 \%$ of the adolescents surveyed who are inclined to consume alcohol, that the use of alcoholic beverages are the substances that are most used in 142, while 55 of them consume tobacco, where they mention that such consumption is a concern to intrafamily problems present in each home, unlike the routine of cannabis linked to anxiety or hyperactivity events that can be the starting point for greater complications in the health of adolescents. Conclusions: The environment that surrounds our youth between 12 and 17 years of age indicates that drug use is increasingly present. Consumption rates have increased in the province of Santa Elena, where the need to develop promotion and prevention campaigns against this social phenomenon is born.
\end{abstract}

Keywords: Adolescents, Consumption, Drugs, Perception, Health.

\section{Introducción}

La dependencia a las sustancias psicoactivas en adolescentes, especialmente las drogas alucinógenas y Psico estimulantes, constituye desde hace unos años un problema de salud pública a nivel mundial, debido a las negativas repercusiones que tiene en ese grupo de edad, incidiendo en la calidad de vida, salud física, salud mental, desarrollo personal y en el desenvolvimiento familiar y social; además 
de que las conductas poco saludables se asocian al consumo de tabaco, alcohol y la falta de actividad física ${ }^{5}$

La última encuesta del Plan Nacional sobre Drogas en jóvenes entre 14 y 18 años en España, "Uso de Drogas en Estudiantes de Secundaria" (ESTUDES 2014-2015), muestra como el consumo de estas constituye una práctica habitual entre los jóvenes de ese país, por tanto, se hace evidente que la realidad actual en relación con este tema es homogénea en todo el mundo y no respeta sexo, continente ni estrato social. ${ }^{6}$

El problema en relación con este tema es que el mismo se agrava cuando se comprueba que los adolescentes tienen una baja percepción de peligro en el uso de algunas de ellas, las de consumo más habitual, como el alcohol, sin que se aprecie un cambio en su forma de pensar en los últimos años a pesar de las estrategias implementadas en los países para tratar de controlar este flagelo. ${ }^{6}$

También la importancia de lograr un control estricto sobre el uso de drogas por los adolescentes radica, en que precisamente en estas edades está ocurriendo aún el pro- ceso de crecimiento y maduración física y psicológica de especial vulnerabilidad, que se pone en riesgo con prácticas como las del uso de drogas.

La Organización Mundial de la Salud define como droga a toda sustancia que, introducida en el organismo vivo, por cualquiera de los medios de administración clásicos o nuevos, es capaz de modificar la actividad del sistema nervioso central y el comportamiento del individuo receptor, así como crear una situación de dependencia o adicción. ${ }^{6}$ El conocimiento que tenga la comunidad científica en relación a la percepción de los adolescentes en relación con el consumo de drogas resulta un factor determinante para la elaboración de estrategias que modifiquen la realidad actual en este sentido, por ese motivo los autores del presente artículo creen prudente y pertinente el desarrollo de la investigación donde se pretende determinar la percepción que tienen los adolescentes de la provincia Santa Elena en relación al consumo de drogas.

\section{Resultados y discusión}

Tabla 1: Niveles de riesgo por sustancias

\begin{tabular}{|l|c|c|c|}
\hline Variables & Nivel de riesgo bajo & Nivel de riesgo moderado & Nivel de riesgo alto \\
\hline Nivel riesgo tabaco & 118 & 17 & 0 \\
\hline Nivel de riesgo bebidas alcohólicas & 110 & 34 & 3 \\
\hline Nivel de riesgo cannabis & 135 & 18 & 1 \\
\hline Nivel de riesgo cocaína & 139 & 3 & 0 \\
\hline Nivel riesgo anfetaminas & 140 & 4 & 0 \\
\hline Nivel de riesgo inhalantes & 129 & 10 & 0 \\
\hline Nivel de riesgo tranquilizantes & 132 & 7 & 0 \\
\hline Nivel de riesgo alucinógenos & 137 & 3 & 0 \\
\hline Nivel de riesgo opiáceos & 135 & 5 & 0 \\
\hline Nivel de riesgo otros & 121 & 4 & 3 \\
\hline
\end{tabular}

Entre la relación de las variables en siguiente tabla, podemos notar que existe en elevado número de adolescentes que se encuentran en un nivel bajo del consumo de sustancias estupefacientes, a diferencia del nivel moderado donde se enmarca un $34 \%$ de los adolescentes encuestados que se inclinan al consumo de alcohol.

Tabla 2: A lo largo de su vida, ¿cuál de las siguientes sustancias ha consumido alguna vez? (Solo para usos no-médicos)

\begin{tabular}{|l|c|c|}
\hline & NO & SI \\
\hline a.Tabaco (cigarrillos, cigarros habanos, tabaco de mascar, pipa, etc. & 353 & 55 \\
\hline b. Bebidas alcohólicas (cerveza, vino, licores, destilados, etc.) & 267 & 142 \\
\hline c. Cannabis (marihuana, costo, hierba, hashish, etc.) & 355 & 48 \\
\hline d. Cocaína (coca, farlopa, crack, base, etc.) & 361 & 39 \\
\hline $\begin{array}{l}\text { e. Anfetaminas u otro tipo de estimulantes (speed, éxtasis, píldoras } \\
\text { adelgazantes, etc.) }\end{array}$ & 359 & 40 \\
\hline
\end{tabular}


Tabla 3: A lo largo de su vida, ¿cuál de las siguientes sustancias ha consumido alguna vez? (Solo para usos nomédicos)...continuacion

\begin{tabular}{|l|l|l|}
\hline f. Inhalantes (colas, gasolina/nafta, pegamento, etc.) & 357 & 46 \\
\hline $\begin{array}{l}\text { g. Tranquilizantes o pastillas para dormir (valium/diazepam, } \\
\text { Trankimazin/Alprazolam/Xanax, Orfidal/Lorazepam, Rohipnol, etc.) }\end{array}$ & 358 & 43 \\
\hline h. Alucinógenos (LSD, ácidos, ketamina, PCP, etc.) & 362 & 39 \\
\hline i. Opiáceos (heroína, metadona, codeína, morfina, dolantina/petidina, etc.) & 360 & 40 \\
\hline j. Otros - especifique: & 309 & 24 \\
\hline
\end{tabular}

Las respuestas expresadas por los adolescentes encuestados muestran que el uso de bebidas alcohólicas son las sustancias que mayor uso realizan, respuesta en 142 adoles- centes a diferencia de 55 adolescentes que consumen tabaco sin control.

Tabla 4: ¿Con qué frecuencia ha consumido las sustancias que ha mencionado en los últimos tres meses, (primera droga, segunda droga, etc.)?

\begin{tabular}{|c|c|c|c|c|c|}
\hline & Nunca & 1 o 2 veces & cada mes & cada semana & $\begin{array}{l}\text { a diario o } \\
\text { casi diario }\end{array}$ \\
\hline $\begin{array}{l}\text { a. Tabaco (cigarrillos, cigarros habanos, } \\
\text { tabaco de mascar, pipa, etc. }\end{array}$ & 205 & 18 & 16 & 0 & 0 \\
\hline $\begin{array}{l}\text { b. Bebidas alcohólicas (cerveza, vino, } \\
\text { licores, destilados, etc.) }\end{array}$ & 140 & 88 & 9 & 1 & 1 \\
\hline $\begin{array}{l}\text { c. Cannabis (marihuana, costo, hierba, } \\
\text { hashish, etc.) }\end{array}$ & 214 & 7 & 0 & 1 & 0 \\
\hline $\begin{array}{l}\text { d. Cocaína (coca, farlopa, crack, base, } \\
\text { etc.) }\end{array}$ & 216 & 2 & 0 & 0 & 0 \\
\hline $\begin{array}{l}\text { e. Anfetaminas u otro tipo de } \\
\text { estimulantes (speed, éxtasis, píldoras } \\
\text { adelgazantes, etc.) }\end{array}$ & 213 & 4 & 0 & 0 & 0 \\
\hline $\begin{array}{l}\text { f. Inhalantes (colas, gasolina/nafta, } \\
\text { pegamento, etc.) }\end{array}$ & 205 & 12 & 3 & 1 & 1 \\
\hline $\begin{array}{l}\text { g. Tranquilizantes o pastillas para dormir } \\
\text { (valium/diazepam, } \\
\text { Trankimazin/Alprazolam/Xanax, } \\
\text { Orfidal/Lorazepam, Rohipnol, etc.) }\end{array}$ & 212 & 6 & 0 & 0 & 0 \\
\hline $\begin{array}{l}\text { h. Alucinógenos (LSD, ácidos, } \\
\text { ketamina, PCP, etc.) }\end{array}$ & 215 & 1 & 0 & 0 & 0 \\
\hline $\begin{array}{l}\text { i. Opiáceos (heroína, metadona, codeína, } \\
\text { morfina, dolantina/petidina, etc.) }\end{array}$ & 214 & 1 & 0 & 0 & 2 \\
\hline j. Otros - especifique: & 210 & 1 & 1 & 0 & 0 \\
\hline
\end{tabular}

Las respuestas obtenidas en esta tabla señalan que el consumo de sustancias en estos tres últimos meses los adolescentes consumen bebidas alcohólicas de 1 a 2 veces por mes sin que sus padres tengan conocimiento y que los demás consumos de sustancias lo realizan en reuniones con compañeros sin presencia de adultos o alguien los controlen.

Tabla 5: Consumo de drogas problemas de salud, sociales, legales y económicos

\begin{tabular}{|c|c|c|c|c|c|}
\hline & Nunca & $\begin{array}{l}1 \text { o } 2 \\
\text { veces }\end{array}$ & $\begin{array}{l}\text { cada } \\
\text { mes }\end{array}$ & $\begin{array}{c}\text { cada } \\
\text { semana }\end{array}$ & $\begin{array}{l}\text { a diario o casi } \\
\text { diario }\end{array}$ \\
\hline $\begin{array}{l}\text { a. Tabaco (cigarrillos, cigarros habanos, tabaco de mascar, } \\
\text { pipa, etc. }\end{array}$ & 182 & 5 & 11 & 1 & 0 \\
\hline
\end{tabular}


Tabla 5: Consumo de drogas problemas de salud, sociales, legales y económicos... continuación

\begin{tabular}{|c|c|c|c|c|c|}
\hline & Nunca & $\begin{array}{l}102 \\
\text { veces }\end{array}$ & $\begin{array}{l}\text { cada } \\
\text { mes }\end{array}$ & $\begin{array}{c}\text { cada } \\
\text { semana }\end{array}$ & $\begin{array}{c}\text { a diario o casi } \\
\text { diario }\end{array}$ \\
\hline $\begin{array}{l}\text { b. Bebidas alcohólicas (cerveza, vino, licores, destilados, } \\
\text { etc.) }\end{array}$ & 164 & 30 & 2 & 2 & 0 \\
\hline c. Cannabis (marihuana, costo, hierba, hashish, etc.) & 179 & 3 & 1 & 0 & 1 \\
\hline d. Cocaína (coca, farlopa, crack, base, etc.) & 183 & 0 & 0 & 0 & 0 \\
\hline $\begin{array}{l}\text { e. Anfetaminas u otro tipo de estimulantes (speed, éxtasis, } \\
\text { píldoras adelgazantes, etc.) }\end{array}$ & 182 & 1 & 0 & 0 & 0 \\
\hline f. Inhalantes (colas, gasolina/nafta, pegamento, etc.) & 179 & 4 & 2 & 0 & 1 \\
\hline $\begin{array}{l}\text { g. Tranquilizantes o pastillas para dormir } \\
\text { (valium/diazepam, Trankimazin/Alprazolam/Xanax, } \\
\text { Orfidal/Lorazepam, Rohipnol, etc.) }\end{array}$ & 182 & 2 & 0 & 0 & 0 \\
\hline h. Alucinógenos (LSD, ácidos, ketamina, PCP, etc.) & 182 & 0 & 0 & 0 & 0 \\
\hline $\begin{array}{l}\text { i. Opiáceos (heroína, metadona, codeína, morfina, } \\
\text { dolantina/petidina, etc.) }\end{array}$ & 182 & 0 & 0 & 0 & 0 \\
\hline j. Otros - especifique: & 178 & 0 & 0 & 0 & 0 \\
\hline
\end{tabular}

La tabla señala que el consumo de sustancias estupecientes en los adolescentes son atribuidas al uso de bebidas alcohólicas concerniente a los problemas intrafamiliares presentes en cada hogar expresado por los adolescentes a diferencia del consumo de cannabis coligado a la ansiedad o eventos de hiperactividad que pueden ser punto de partida para mayores complicaciones en la salud de los adolescentes.

Tabla 6: ¿Un amigo, un familiar o alguien más alguna vez ha mostrado preocupación por su consume de (primera droga, segunda droga, etc.)?

\begin{tabular}{|c|c|c|c|}
\hline & no, nunca & $\begin{array}{l}\text { sí, en los últimos } 3 \\
\text { meses }\end{array}$ & $\begin{array}{l}\text { sí, pero no en los } \\
\text { últimos } 3 \text { meses }\end{array}$ \\
\hline $\begin{array}{l}\text { a. Tabaco (cigarrillos, cigarros habanos, tabaco de mascar, } \\
\text { pipa, etc. }\end{array}$ & 165 & 5 & 3 \\
\hline c. Cannabis (marihuana, costo, hierba, hashish, etc.) & 160 & 6 & 4 \\
\hline $\begin{array}{l}\text { e. Anfetaminas u otro tipo de estimulantes (speed, éxtasis, } \\
\text { píldoras adelgazantes, etc.) }\end{array}$ & 165 & 1 & 2 \\
\hline f. Inhalantes (colas, gasolina/nafta, pegamento, etc.) & 163 & 4 & 4 \\
\hline $\begin{array}{l}\text { g. Tranquilizantes o pastillas para dormir (valium/diazepam, } \\
\text { Trankimazin/Alprazolam/Xanax, Orfidal/Lorazepam, } \\
\text { Rohipnol, etc.) }\end{array}$ & 163 & 3 & 2 \\
\hline $\begin{array}{l}\text { i. Opiáceos (heroína, metadona, codeína, morfina, } \\
\text { dolantina/petidina, etc.) }\end{array}$ & 164 & 3 & 1 \\
\hline j. Otros - especifique: & 163 & 1 & 2 \\
\hline
\end{tabular}

Por medio de la aplicación de este recuadro se puede identificar que todos los adolescentes encuestados coinciden que sus familiares y amigos más cercano no se interesan por conocer si consumen o no, estos resultados nos permiten hacer una reflexión en la investigación llevando a la importancia de realizar programas de concientización en los adolescentes dado el riesgo que conlleva el uso de sustancias estupefacientes. 


\section{Conclusiones}

Dentro de la prevención del consumo de sustancias como drogas, alcohol y tabaco, consideramos que la información sobre las drogas es un elemento imprescindible y de gran eficacia para disminuir el consumo de aquellos que, lamentablemente han iniciado, y evitar que lo hagan los que todavía se mantienen al margen de esta cruda realidad.

En la investigación realizada se evidenció que en los últimos tres meses, los familiares y amigos de los adolescentes encuestados no presentaron ninguna preocupación por el consumo de sustancias siendo las bebidas alcohólicas a la mayor preferencia por los adolescentes en provincia Santa Elena, lo que conlleva a direccionar a los consumidores al uso de otras sustancias.

No todo consumo en un adolescente es problemático, pero sí es imprescindible pensar que todo consumo en esta etapa y en especialmente en adolescentes, es considerado de alto riesgo.

\section{Fuente de Financiamiento}

Este estudio es autofinanciado.

\section{Conflicto de Intereses}

No existen conflictos personales, profesionales, financieroso de otro tipo.

\section{Consentimiento Informado}

Los autores cuentan con el consentimiento informado de los pacientes para la investigación, la publicación del caso y sus imágenes.

\section{Referencias Bibliográficas}

1. Alejandro, M. H. (2011). Consumo dealcohol y drogas en adolescentes. Revista Médica Clínica Las Condes, 98-109.

2. Fergusson, D. M., Boden, J. M., \& Horwood, L. J. (2006). Cannabis use and other illicit drug use: testing the cannabis gateway hypothesis. Addiction (Abingdon, England), 101(4), 556-569. https://doi.org/10.1111/j.13600443.2005.01322.x

3. José P. Espada, K. W. (2003). Adolescencia: consumo de alcohol y otras drogas. Red de Revistas Científicas de América Latina, el Caribe, España y Portuga, pp. 9-17.

4. NACIONAL, C. (2005). Tratamiento del consumo de drogas para niños/as y adolescentes en vulnerabilidad social. Santiago de Chile: Ministerio del Interior, Consejo Nacional para el Control de.

5. Polo-Gallardo R., Rebolledo Cobos R., MendinuetaMartinez M., y Reniz Acosta K. (2017). Consumo de drogas y la práctica de actividad física en adolescentes:revisión narrativa. Revista de la Facultad Ciencias de la Salud. Universidad del Cauca, 19(2), 29-37.

6. Terán Prieto A. (2017). Drogodependencias en el adolescente. Actuación desde la consulta. En: AEPap (ed.). Curso de Actualización Pediatría. Madrid: Lúa Ediciones 3.0. p. 493-501.

Recibido: 15 de julio de 2020

Aceptado: 15 de septiembre de 2020 
